\title{
A Robust Method to Suppress Jamming for GNSS Array Antenna Based on Reconstruction of Sample Covariance Matrix
}

\author{
Yanyun Gong, Ling Wang, Rugui Yao, and Zhaolin Zhang \\ School of Electronics and Information, Northwestern Polytechnical University, Xian 710072, China \\ Correspondence should be addressed to Ling Wang; lingwang@nwpu.edu.cn
}

Received 27 October 2016; Revised 5 January 2017; Accepted 23 January 2017; Published 12 March 2017

Academic Editor: Huapeng Zhao

Copyright (C) 2017 Yanyun Gong et al. This is an open access article distributed under the Creative Commons Attribution License, which permits unrestricted use, distribution, and reproduction in any medium, provided the original work is properly cited.

\begin{abstract}
The Global Navigation Satellite System (GNSS) receiver is vulnerable to active jamming, which results in imprecise positioning. Therefore, antijamming performance of the receiver is always the key to studies of satellite navigation system. In antijamming application of satellite navigation system, if active jamming is received from array antenna main-lobe, main-lobe distortion happens when the adaptive filtering algorithm forms main-lobe nulling. A robust method to suppress jamming for satellite navigation by reconstructing sample covariance matrix without main-lobe nulling is proposed in this paper. No nulling is formed while suppressing the main-lobe jamming, which avoids main-lobe direction distortion. Meanwhile, along with adaptive pattern control (APC), the adaptive pattern of array antenna approaches the pattern without jamming so as to receive the matching navigation signal. Theoretical analysis and numerical simulation prove that this method suppresses jamming without main-beam distortion. Furthermore, the output SINR will not decrease with the main-lobe distortion by this method, which improves the antijamming performance.
\end{abstract}

\section{Introduction}

Satellite navigation system takes advantage of navigating satellites for positioning and navigation [1]. It plays a vital role in transportation management, time service, search and rescue, and geodesy due to its globalized and full-time duration as well as precision [2-6]. Nowadays, GNSS service includes Chinese Beidou Navigation Satellite System (BDS), American Global Positioning System (GPS), and Russian's GLONASS [7].

As the navigation satellites operate on the orbit of $20200 \mathrm{~km}$ far away from the Earth surface, the signal emitted by them that is finally received by the GNSS user becomes quite weak $[8,9]$. Under this circumstance, navigation signal is vulnerable to active jamming, which leads to the loss of navigation signal. Therefore, the antijamming performance is a prerequisite for the navigation system.

Adaptive spatial filtering technology is one of the main antijamming techniques for GNSS as well as a major branch of adaptive array signal processing [10]. Embedding GPS antijamming technology based on power inversion algorithm is proposed which does not require a prior information of navigation signal nor array arrangement [11]. GPS antijamming methods based on spatial time adaptive processing (STAP) increase the tapping of signal's time domain so that the antijamming performance is improved [12]. An enhanced short-time Fourier transform (STFT) jamming mitigation system is proposed in [13] which employs several windows to increase time-frequency plane resolution.

However, the above-listed methods do not constrain the receiving of useful navigation satellite signal, while only nulling the jamming signal. Therefore, it is not guaranteed that the pattern's main-lobe points to the direction of satellite signal, which posts negative influence on the receiving navigation signal [14]. A robust antijamming algorithm is proposed under this circumstance. Relevant documents take advantage of minimum variance distortionless response (MVDR) to suppress jamming on GPS. This algorithm constrains the antenna pattern and improves the antijamming performance [15]. Then multiple constrained minimum variance-spatial temporal adaptive processing (MCMV-STAP) was proposed in [16] to enhance the desired 


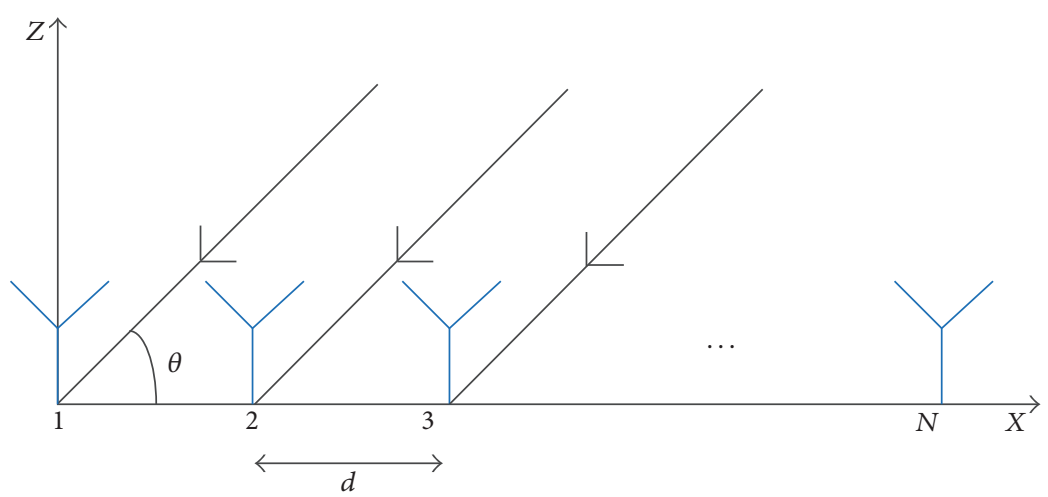

FIgURE 1: The linear antenna array geometry.

satellite signals and suppress jamming, which can broaden the width of nulls and provide approximately flat gains in other directions.

However, this useful algorithm still forms nulling when active jamming exists in the pattern's main-lobe, which leads to increased distortion and decreased SINR. Thus, the receiver cannot use navigation signal for positioning [17].

A robust method to suppress jamming for satellite navigation by reconstructing sample covariance matrix is proposed in this paper. In this method, adaptive beamforming and antijamming are achieved by combining APC with linearly constrained minimum variance (LCMV) beamformer [18-20]. In proposed method, sample covariance matrix is decomposed first; then jamming components of mainlobe are distinguished by the correlation of eigenvector and desired signal steering vector. The main-lobe jamming eigenvectors are calculated by the correlation between mainlobe jamming eigenvectors and desired signal steering vector. The sampling covariance matrix is reconstructed to eliminate the influence of the main-lobe jamming component on the pattern. Eigen-projection matrix can be constructed after eigenvector of main-lobe jamming is acquired so as to eliminate main-lobe jamming components; thus, main-lobe jamming is suppressed. When suppressing all jamming, the pattern only forms nulling in the side-lobe. Then APC is processed to constrain the weight of the LCMV beamformer so that adaptive pattern approaches the pattern without jamming further.

When the main-lobe jamming is suppressed, the proposed method does not form nulling in the main-lobe jamming direction, so that the beam direction will not be offset due to the main-lobe antijamming. This ensures that the beam will point to the navigation satellite still.

Therefore, the output SINR of the satellite navigation signal received by the antenna array in the direction of the navigation satellite is higher than that of other methods relatively. The output SINR is guaranteed, it will be easier to calculate the satellite navigation signal after suppressing the jamming for the GNSS receiver. Under the premise of satisfying the tracking sensitivity of satellite navigation receiver, the proposed algorithm in this paper can suppress more jamming than other algorithms.
Theoretical analysis and numerical simulation prove that the direction of the pattern is not offset by the suppression of main-lobe jamming. The output SINR of satellite navigation signal will not decrease due to the directional offset of the pattern, and the output SINR is only related to the intensity of the input jamming signal, which will be helpful for the navigation receiver to process the navigation signal.

This paper is organized as follows. The array model and signal's mathematic model are provided in Section 2. The details of the proposed method as well as theoretical analysis on reconstruction of covariance matrix and APC are developed in Section 3. Simulations are carried out, which include analysis on the adaptive pattern and the output SINR in Section 4. Section 5 shows the conclusion.

\section{Problem Formulation}

2.1. Signal Model. Suppose an $N$-element uniform linear array which is showed in Figure $1, d$ is the distance between array elements, and all the array elements are homogeneous and isotropic.

There is a far-field desired signal and $P$ narrowband jamming signals incident as plane wave (wavelength $\lambda$ ) [21]. The desired signal and the jamming-signal angles of arrival are $\theta_{0}$ and $\theta_{j}(j=1,2, \ldots, P)$; then the signal received by the array can be expressed as

$$
\mathbf{X}(k)=\mathbf{A S}(k)+\mathbf{n}(k),
$$

where $\mathbf{X}(k)$ refers to the matrix of array received data,

$$
\mathbf{X}(k)=\left[\mathbf{x}_{1}(k), \mathbf{x}_{2}(k), \ldots, \mathbf{x}_{N}(k)\right],
$$

and $\mathbf{A}$ refers to array steering vector, which is merely relevant to signal incidence angle and can be expressed as

$$
\mathbf{A}=\left[\mathbf{a}\left(\theta_{0}\right), \mathbf{a}\left(\theta_{1}\right), \ldots, \mathbf{a}\left(\theta_{P}\right)\right]^{T} .
$$

In (3),

$$
\mathbf{a}\left(\theta_{k}\right)=\left[1, e^{i \beta_{k}}, \ldots, e^{i(N-1) \beta_{k}}\right]^{T}, \quad(k=0,1, \ldots, P)
$$

which is the array steering vector corresponding to the signal source; meanwhile, $\beta_{k}=(2 \pi / \lambda) d \sin \theta_{k}$. 


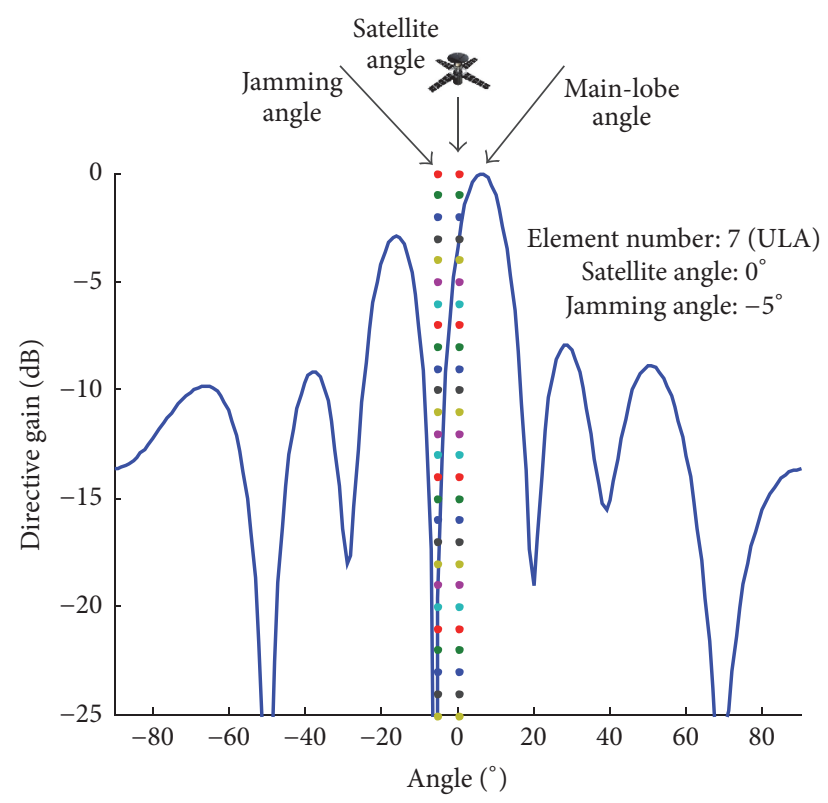

FIGURE 2: Effect of main-lobe jamming on satellite signal reception.

$\mathbf{n}(k)$ in (1) stands for noise matrix and $\mathbf{S}(k)$ for signal matrix; the latter one includes the navigation signal $\mathbf{s}_{0}(k)$ and the jamming signal $\mathbf{s}_{j}(k)$. So $\mathbf{X}(k)$ can be expressed as

$$
\mathbf{X}(k)=\mathbf{a}\left(\theta_{0}\right) \mathbf{s}_{0}(k)+\sum_{j=1}^{P} \mathbf{a}\left(\theta_{j}\right) \mathbf{s}_{j}(k)+\mathbf{n}(k),
$$

and sample covariance matrix can be reached as

$$
\mathbf{R}_{X}=E\left[\mathbf{X}(t) \mathbf{X}^{H}(t)\right]
$$

where $E[*]$ and $(*)^{H}$ denote the expectation operator, respectively, and conjugate transpose.

2.2. The $L C M V$ Beamformer. The gain constraint by the LCMV beamformer in the expected signal direction is 1 (unit constraint), and the array output power is minimized, in which jamming and noise decrease is suppressed [22]. Weight vector of LCMV beamformer can be reached by this equation

$$
\begin{array}{ll}
\min _{W} & \mathbf{W}^{H} \mathbf{R}_{X} \mathbf{W}, \\
\text { s.t. } & \mathbf{W}^{H} \mathbf{C}=\mathbf{F}^{H},
\end{array}
$$

in which $\mathbf{C}$ is the constraint matrix and $\mathbf{F}$ is the corresponding response constraint vector. The weight vector of LCMV beamformer can be expressed as

$$
\mathbf{W}_{\mathrm{LCMV}}=\mathbf{R}_{X}^{-1} \mathbf{C}\left(\mathbf{C}^{H} \mathbf{R}_{X}^{-1} \mathbf{C}\right)^{-1} \mathbf{F}
$$

in which $\mathbf{W}_{\mathrm{LCMV}}$ is the weight vector of LCMV beamformer [23].

When LCMV beamformer is utilized to suppress mainlobe jamming -5 degrees, its pattern is shown in Figure 2. The main-lobe already distorts from the expected angle of the satellite signal when suppressing this jamming signal. The output SINR decreases; meanwhile, the nulling position is not precise.

Therefore, in the application of satellite navigation antijamming, both the performance of the interference suppression algorithm and the pattern distortion lead to the loss of positioning function of the receiver prematurely. In the process of suppressing the main-lobe jamming, the pattern distortion also seriously affects the reception of satellite navigation signals. The output SINR of satellite navigation signal is affected by the input jamming-signal intensity and the directional pattern offset. When the output signal to noise ratio of the array is decreased, the performance of the antijamming degrades. This paper focuses on the avoidance of pattern distortion in GNSS antijamming, so that the output signal is only affected by the input jamming intensity.

\section{Proposed Method}

This section proposes a new antijamming method by overcoming the shortcoming of LCMV beamformer in GNSS antijamming in Section 2. This method can deal with the distortion of pattern direction when suppressing main-lobe jamming. The adaptive pattern approaches the pattern without jamming so as to effectively receive navigation signal.

Since the navigation signal received by the antenna array is weak in power, this signal can be deemed to be composed of jamming and noise. So sample covariance matrix can be considered to be made up of jamming-and-noise covariance matrix. It meets the requirements of robust beamforming. Therefore, to prevent pattern distortion, coping with mainlobe jamming is the only step to be paid attention to. The detailed process is described as follows.

3.1. Covariance Matrix Reconstruction. The eigen-decomposition of a sample covariance matrix can be expressed as

$$
\mathbf{R}_{X}=\sum_{i=1}^{N} \lambda_{i} \boldsymbol{\tau}_{i} \boldsymbol{\tau}_{i}^{H}=\boldsymbol{\Gamma}_{s} \boldsymbol{\Lambda}_{s} \boldsymbol{\Gamma}_{s}{ }^{H}+\boldsymbol{\Gamma}_{n} \boldsymbol{\Lambda}_{n} \boldsymbol{\Gamma}_{n}{ }^{H}
$$

where $\lambda_{1} \geq \lambda_{2} \geq \cdots \geq \lambda_{p} \geq \lambda_{P+1}=\cdots=\lambda_{N}=\sigma_{n}^{2}$ refers to descending-order eigenvalue of $\mathbf{R}_{X} \cdot \boldsymbol{\Gamma}_{s}=\left[\boldsymbol{\tau}_{1}, \ldots, \boldsymbol{\tau}_{P}\right]$ and $\boldsymbol{\Gamma}_{n}=\left[\boldsymbol{\tau}_{P+1}, \ldots, \boldsymbol{\tau}_{N}\right]$ are signal-and-jamming subspace and noise subspace, respectively, among which $\boldsymbol{\tau}_{i}$ is the eigenvector corresponding to $\lambda_{i} . \Lambda_{s}=\operatorname{diag}\left\{\lambda_{1}, \ldots, \lambda_{p}\right\}$ and $\Lambda_{n}=$ $\operatorname{diag}\left\{\lambda_{p+1}, \ldots, \lambda_{N}\right\}$ are the diagonal matrices of $\boldsymbol{\Gamma}_{s}$ and $\boldsymbol{\Gamma}_{n}$, respectively [24].

Eigenvalue and eigenvector of the main-lobe jamming signal can be achieved by the correlation of decomposed sample covariance matrix and steering vector of signal's incidence angle [25]. The correlation coefficient equation between $\mathbf{a}_{1}$ and $\mathbf{a}_{2}$ is

$$
\operatorname{cor}\left(\mathbf{a}_{1}, \mathbf{a}_{2}\right)=\frac{\left|\mathbf{a}_{1}{ }^{H} \mathbf{a}_{2}\right|}{\left\|\mathbf{a}_{1}\right\|\left\|\mathbf{a}_{2}\right\|} .
$$

The correlation coefficient between steering vector of incidence signal $\mathbf{a}\left(\theta_{s}\right)$ and eigenvectors of various-angle jamming $\boldsymbol{\Gamma}_{s}=\left[\boldsymbol{\tau}_{1}, \ldots, \boldsymbol{\tau}_{P}\right]$ can also be achieved by (10). 


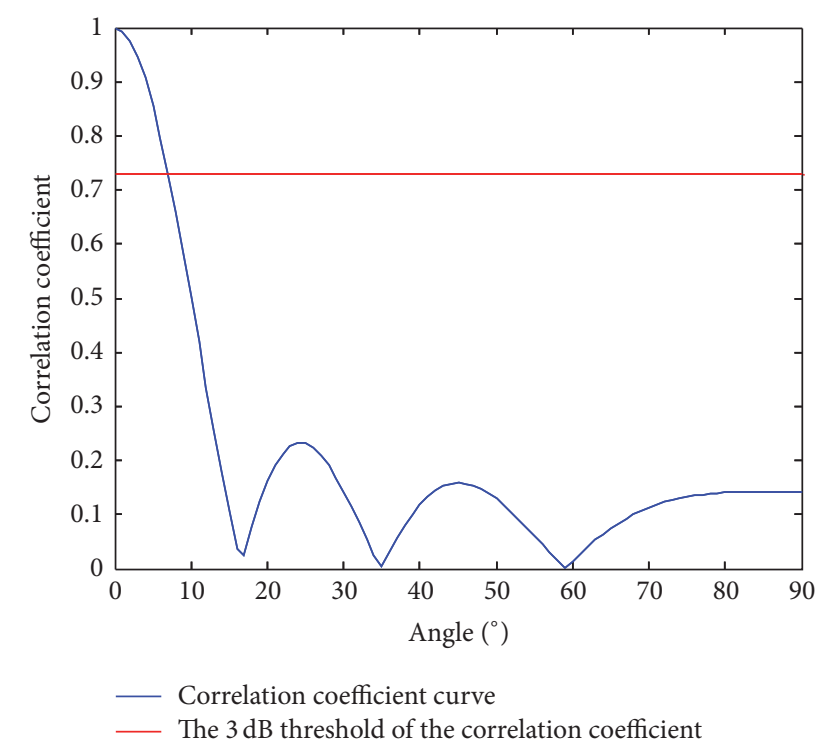

FIGURE 3: Correlation coefficient between various-angle steering vector and 0 degree steering vector.

Figure 3 shows the curve of correlation coefficient between various-angle steering vectors and the steering vector at 0 degrees based on the seven-element ULA structure.

This figure shows that the correlation coefficient curve descends as the angle increases. Taking advantage of this character, this paper assumes an interval of correlation coefficient $(\varepsilon, 1)$, among which $\varepsilon$ can be reached by arrayelement structure.

The constant value in Figure 3 is the corresponding coefficient of main-lobe boundary angle reached by the seven-element uniform linear array. Main-lobe width can be defined as the $3 \mathrm{~dB}$ bandwidth. Eigenvector in this range can be deemed as main-lobe eigenvector of jamming eigenvector.

Therefore, it can be assumed that this steering vector is the one corresponding to main-lobe jamming when the steering vector of incident signal and coefficient of jamming eigenvector are within the interval $(\varepsilon, 1)$. This process can be expressed as

$$
\varepsilon \leq \operatorname{cor}\left(\boldsymbol{\tau}_{i}, \mathbf{a}\left(\theta_{s}\right)\right) \leq 1, \quad\left(\boldsymbol{\tau}_{i} \in \Gamma_{s}\right),
$$

among which $\Gamma_{s}=\left[\boldsymbol{\tau}_{1}, \ldots, \boldsymbol{\tau}_{P}\right]$ is the eigenvector of the various-angle jamming, and

$$
\operatorname{cor}\left(\boldsymbol{\tau}_{i}, \mathbf{a}\left(\theta_{s}\right)\right)=\frac{\left\|\boldsymbol{\tau}_{i}{ }^{H} \mathbf{a}\left(\theta_{s}\right)\right\|}{\left\|\boldsymbol{\tau}_{i}\right\|\left\|\mathbf{a}\left(\theta_{s}\right)\right\|} .
$$

Eigenvector and eigenvalue of main-lobe jamming $\widehat{\boldsymbol{\tau}}_{i}$ and $\widehat{\boldsymbol{\tau}}_{i}$ can be decided by (11).

Eigenvalue analysis tells that the array response of adaptive beamformer can be expressed as the difference of no jamming response and jamming beam response [26]

$$
\begin{aligned}
\mathbf{F}(\theta) & =\frac{\mu}{\sigma_{n}^{2}}\left\{\mathbf{a}^{H}\left(\theta_{s}\right) \mathbf{a}(\theta)\right. \\
& \left.-\sum_{i=1}^{N} \frac{\lambda_{i}-\sigma_{n}^{2}}{\lambda_{i}}\left[\boldsymbol{\tau}_{i} \mathbf{a}\left(\theta_{s}\right)\right] \boldsymbol{\tau}_{i}^{H} \mathbf{a}(\theta)\right\},
\end{aligned}
$$

in which $\mathbf{F}(\theta)$ is the array response of adaptive beamformer, and

$$
\mu=\frac{1}{\mathbf{a}^{H}\left(\theta_{s}\right) \widehat{\mathbf{R}}^{-1} \mathbf{a}\left(\theta_{s}\right)} .
$$

Meanwhile, the noise power can be estimated by the mean of $N-P$ smaller eigenvalues, which can be expressed as

$$
\sigma_{n}^{2}=\frac{\lambda_{P+1}+\cdots+\lambda_{N}}{N-P}
$$

Generally speaking, jamming power is considerably greater than the noise power. Therefore, as the eigenvalue corresponding to jamming signal, $\lambda_{i}$ overwhelms $\sigma_{n}^{2}$. So this equation is derived

$$
\frac{\lambda_{i}-\sigma_{n}^{2}}{\lambda_{i}} \approx 1, \quad i \in(1, \ldots, P) .
$$

That is how the adaptive array response forms nulling in the jamming direction for jamming suppression [18]. It does not form the nulling when $\lambda_{i}$ corresponds to noise eigenvalue.

$\widehat{\lambda_{i}}$ contains all power of the main-lobe jamming. If the eigenvalue of the main-lobe jamming is reduced to the noise power, the adaptive weight vector will not form a nulling in the main-lobe jamming direction when the adaptive weight vector is calculated.

Therefore, when eigenvalue of main-lobe jamming $\widehat{\lambda_{i}}$ is replaced by the mean of noise eigenvalue, which results in that the eigenvalue of main-lobe jamming decreases to background noise, the replaced expression is

$$
\widehat{\lambda_{i}}=\sigma_{n}^{2}=\frac{\lambda_{P+1}+\cdots+\lambda_{N}}{N-P} .
$$

In order to deal with noise in the adaptive algorithm, $\Lambda_{n}$ is also replaced by the average value of noise. The reconstructed eigenvalue matrix of $\boldsymbol{\Lambda}_{n}$ can be expressed as

$$
\widehat{\Lambda}=\operatorname{diag}\left\{\lambda_{1}, \ldots, \widehat{\lambda_{i}}, \ldots, \lambda_{p}, \sigma_{n}^{2}, \ldots, \sigma_{n}^{2}\right\} .
$$

The steering vector matrix makes no change, so the reconstructed sample covariance matrix $\widetilde{\mathbf{R}}_{r}$ can be expressed as

$$
\widetilde{\mathbf{R}}_{r}=\Gamma \widehat{\boldsymbol{\Lambda}} \boldsymbol{\Gamma}^{H}
$$

where $\boldsymbol{\Gamma}=\left[\boldsymbol{\Gamma}_{s}, \boldsymbol{\Gamma}_{n}\right]$ and $\widetilde{\mathbf{R}}_{r}$ excludes main-lobe jamming components. LCMV beamforming adaptive weight value can be calculated as

$$
\widetilde{\mathbf{W}}_{\mathrm{LCMV}}=\widetilde{\mathbf{R}}_{r}^{-1} \mathbf{C}\left(\mathbf{C}^{H} \widetilde{\mathbf{R}}_{r}^{-1} \mathbf{C}\right)^{-1} \mathbf{F} .
$$

Adaptive array antenna pattern produces no jamming nulling in main-lobe while suppressing main-lobe jamming. 
3.2. Adaptive Pattern Control. The weight value of LCMV beamformer computed from the reconstructed jammingand-noise covariance matrix forms no nulling when suppressing main-lobe jamming and no pattern distortion appears. However, mistakes still appear in adaptive aerial pattern because of other elements. Under this circumstance, APC can be utilized to achieve the pattern without jamming so as to make adaptive management more robust.

When experiment data contains merely independent white noise, the array covariance matrix is the unit matrix multiplied by the noise power. Then the weight vector without jamming of LCMV can be expressed as

$$
\mathbf{W}_{q}=\mathbf{C}\left(\mathbf{C}^{H} \mathbf{C}\right)^{-1} \mathbf{F}
$$

In order to adjust adaptive pattern to the pattern without jamming, The weight vector $\mathbf{W}_{q}$ without jamming of the LCMV is constrained to be $\overline{\mathbf{W}}_{q}$, which meets the constraint $\mathbf{W}^{H} \mathbf{C}=\mathbf{H}^{H}$ and approaches the weight vector without jamming $\mathbf{W}_{q} \cdot \overline{\mathbf{W}}_{q}$ is considered to be the solution to the following constrained least squares fitting:

$$
\begin{gathered}
\overline{\mathbf{W}}_{q}=\arg \min \quad\left\{\left(\overline{\mathbf{W}}-\mathbf{W}_{q}\right)^{H}\left(\overline{\mathbf{W}}-\mathbf{W}_{q}\right)\right\}, \\
\text { s.t. } \quad \overline{\mathbf{W}} \mathbf{C}=\mathbf{F}^{H},
\end{gathered}
$$

where $\mathbf{W}_{q}{ }^{H} \mathbf{a}\left(\theta_{0}\right)=1$.

After Lagrange multiplier method is utilized to solve the following expression, the revised weight vector without jamming $\overline{\mathbf{W}}_{q}$ is

$$
\overline{\mathbf{W}}_{q}=\left[\mathbf{I}-\mathbf{C}\left(\mathbf{C}^{H} \mathbf{C}\right)^{-1} \mathbf{C}\right] \mathbf{W}_{q}+\mathbf{C}\left(\mathbf{C}^{H} \mathbf{C}\right)^{-1} \mathbf{F} .
$$

Supposing $\mathbf{Z}$ is the block matrix of constraint matrix $\mathbf{C}$ and $\mathbf{Z C}=0$, so

$$
\overline{\mathbf{W}}_{q}=\mathbf{Z}^{H}\left(\mathbf{Z Z}^{H}\right)^{-1} \mathbf{Z} \mathbf{W}_{q}+\mathbf{C}\left(\mathbf{C}^{H} \mathbf{C}\right)^{-1} \mathbf{F}
$$

if $\mathbf{W}_{Z}=\mathbf{Z}^{H}\left(\mathbf{Z Z} \mathbf{Z}^{H}\right)^{-1} \mathbf{W}_{0}$, let $\overline{\mathbf{C}}=\left[\mathbf{C}, \mathbf{W}_{Z}\right]$ and $\overline{\mathbf{F}}=\left[\begin{array}{c}\mathbf{F} \\ \left\|\mathbf{W}_{Z}\right\|^{2}\end{array}\right]$.

So the revised the weight vector without jamming $\overline{\mathbf{W}}_{q}$ becomes $\overline{\mathbf{W}}_{q}=\overline{\mathbf{C}}\left(\overline{\mathbf{C}}^{H} \overline{\mathbf{C}}\right)^{-1} \overline{\mathbf{F}}$.

Finally, according to formula (8), the LCMV weight vector can be revised as

$$
\widehat{\mathbf{W}}_{\mathrm{LCMV}}=\widetilde{\mathbf{R}}_{r}^{-1} \overline{\mathbf{C}}\left(\overline{\mathbf{C}}^{H} \widetilde{\mathbf{R}}_{r}^{-1} \overline{\mathbf{C}}\right)^{-1} \overline{\mathbf{F}}
$$

3.3. Array Antenna Output. Eigen-projection matrix can be constructed after the eigenvector of main-lobe jamming is acquired so as to eliminate main-lobe jamming components. Eigenvector of main-lobe jamming $\widehat{\boldsymbol{\tau}}_{i}$ is the prerequisite for this construction.

$$
\mathbf{B}=\mathbf{I}-\widehat{\boldsymbol{\tau}}_{i}\left(\widehat{\boldsymbol{\tau}}_{i}^{H} \widehat{\boldsymbol{\tau}}_{i}\right)^{-1} \widehat{\boldsymbol{\tau}}_{i}^{H},
$$

in which $\mathbf{B}$ is the eigen-projection matrix.
The eigen-projection matrix can be utilized for pretreating echo data $\mathbf{X}$ in order to eliminate main-lobe jamming part in the signal.

$$
\mathbf{X}_{b}=\mathbf{B X}
$$

where $\mathbf{X}_{b}$ refers to the output of the pretreatment, made up of navigation signal, jamming signal, and noise. Equation (27) can get rid of main-lobe jamming but not side-lobe one. This data requires adaptive weight treatment,

$$
\mathbf{Y}=\widehat{\mathbf{W}}_{\text {LCMV }}^{H} \mathbf{B X} \text {. }
$$

After that, remaining side-lobe jamming is suppressed. Until then jamming on array output signal is completely under control, and no main-lobe nulling appears. Therefore, a relatively higher output SINR is acquired.

The flowchart of the proposed method is shown in the green frame in Figure 4.

The jamming suppression processing steps are shown as follows:

(1) The sample covariance matrix of signals received by the array antenna is decomposed and the correlation is calculated. So the main-lobe jamming eigenvector $\widehat{\boldsymbol{\tau}}_{i}$ is calculated.

(2) Reconstructed sample covariance matrix $\widetilde{\mathbf{R}}_{r}$ excludes main-lobe jamming components, eliminating the effect of main-lobe jamming on pattern. At the same time, eigen-projection matrix $\mathbf{B}$ can be constructed after the eigenvector of the main-lobe jamming is acquired so as to eliminate the main-lobe jamming components and the main-lobe jamming can be suppressed.

(3) Use LCMV to calculate antijamming weight value $\widetilde{\mathbf{W}}_{\text {LCMV }}$. Since $\widetilde{\mathbf{R}}_{r}$ does not contain the main-lobe jamming component, the pattern corresponding to $\widetilde{\mathbf{W}}_{\text {LCMV }}$ does not form nulls at the main-lobe jamming.

(4) Use APC to make beam pattern approach the pattern without jamming, calculating the final beamformer weight value $\widehat{\mathbf{W}}_{\mathrm{LCMV}}$.

(5) Calculate beamforming output $\mathbf{Y}=\widehat{\mathbf{W}}_{\text {LCMV }}^{H} \mathbf{B X}$. The adaptive pattern not only suppresses the jamming but also does not form a nulling in the main-lobe jamming.

\section{Simulation Analysis}

In this section, five experiments are listed to illustrate the performance of this robust method for jamming suppression. GPS navigation signals are all on frequency point L1 and the input SNR is $-20 \mathrm{~dB}$ [27].

This paper is written with these assumptions: jamming comes from point source. Geometrical aperture is far less than the aerial-radiation distance. Signal received by array is plane wave. Transmission medium is equivalently isotropic 


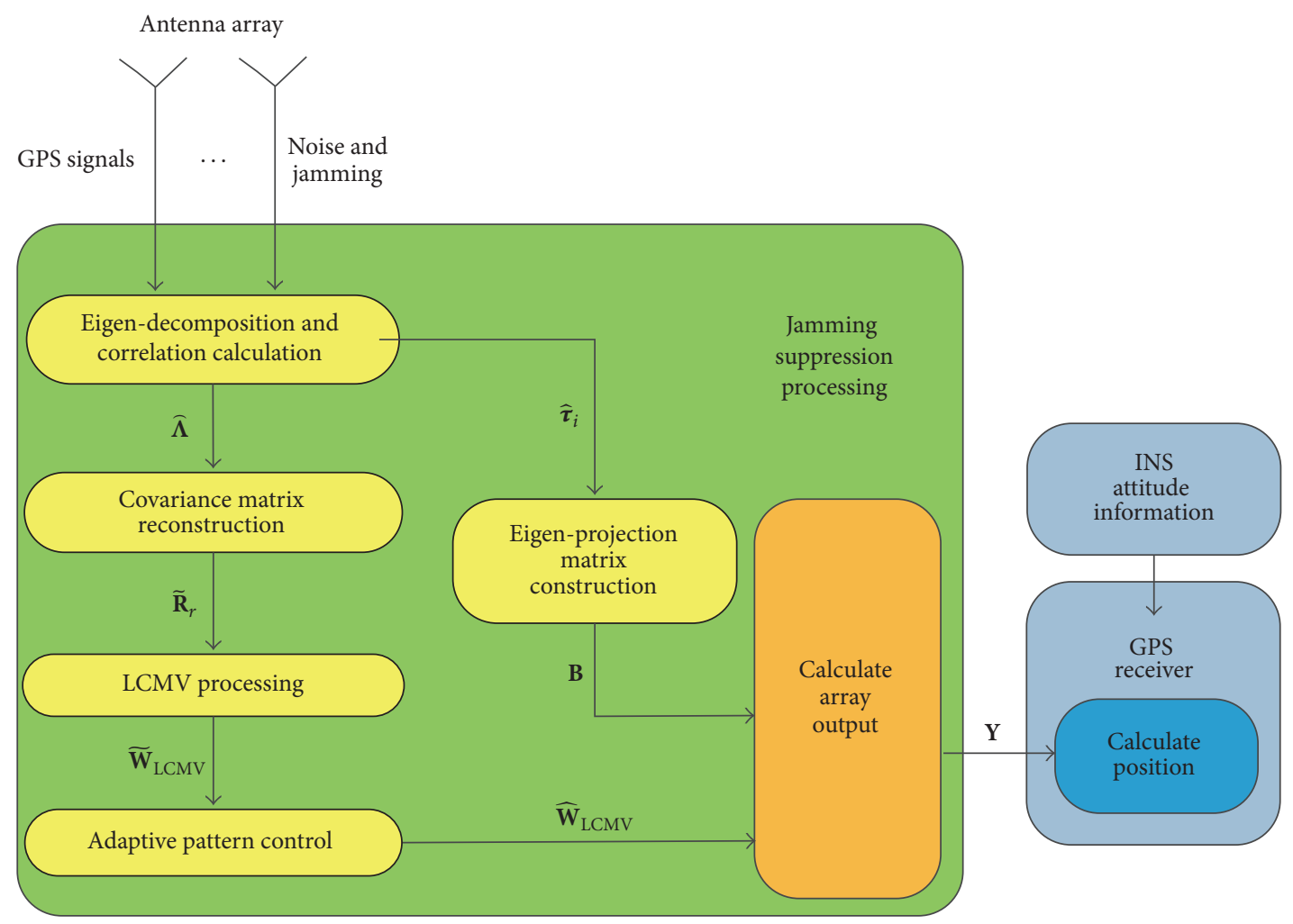

FIgURE 4: The flowchart of the proposed method.

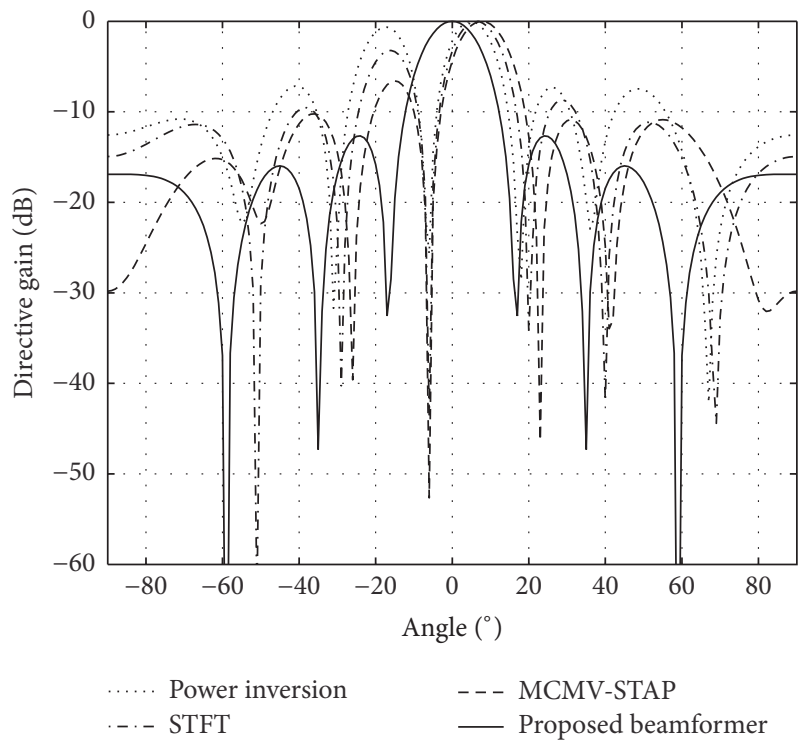

FIGURE 5: The array antenna pattern of antijamming for different method: the angle of arrival of the satellite signal is 0 degrees and the angle of arrival of jamming signal is -6 degrees.

and uniform all around. The zero-mean noise is robust, random, and of no relation with expected signal.

Power inversion algorithm is utilized for performance comparison, in which STFT algorithm (based on spacefrequency structure) and MCMV-STAP algorithm (based on space-time structure) are the two parts [28]. The number of snapshots is 500 throughout the simulation, and all results are the mean of 100 Monte Carlo experiment.

Table 1 shows angles of navigation signal-and-jamming signal. Each scenario of the simulation chooses satellite and jamming signals of different (incidence) angles.

\subsection{Influence of Jamming-Signal Incidence Angle on Adaptive} Pattern. In this simulation experiment, antenna array uses uniform linear array (ULA) with seven-array elements, with half wavelength width as the distance between array elements.

Figure 5 takes the first scene in Table 1 for simulation: presuming that incidence angle of satellite signal is 0 degrees. Jamming signal has an incidence angle of -6 degrees and an ISR of $50 \mathrm{~dB}$ and produces main-lobe jamming to sevenarray antenna. So the algorithm in this paper forms no mainlobe nulling, while the other three algorithms form nulling at -6 degrees. As a result, algorithms of minimum power, STFT, and MCMV-STAP lead to deviation of beam direction: 6 degrees for the first but more on side-lobe and 8 degrees for the last but less on side-lobe. Since no jamming nulling exists in this paper's algorithm, main-lobe does not distort, and the pattern approaches the pattern without jamming as a whole.

Figure 6 shows the simulation in the second scene of Table 1: presuming that incidence angle of satellite signal is 0 degrees. Incidence angles of jamming signal are -6 degrees and 12 degrees with an ISR of $50 \mathrm{~dB}$. Although the algorithms of minimum power, STFT, and MCMV-STAP can precisely produce nulling in jamming position, beam direction already 
TABLE 1: Satellite and Jamming Scenarios.

\begin{tabular}{|c|c|c|c|c|c|c|c|c|}
\hline Signal & Angle & Scenario 1 & Scenario 2 & Scenario 3 & Scenario 4 & Scenario 5 & Scenario 6 & Scenario 7 \\
\hline Satellite & 0 degrees & $\bullet$ & $\bullet$ & $\bullet$ & & & & \\
\hline Satellite & -20 degrees & & & & $\bullet$ & & & \\
\hline Satellite & 20 degrees & & & & & - & & \\
\hline Satellite & 70 degrees & & & & & & $\bullet$ & \\
\hline Satellite & 10 degrees & & & & & & & $\bullet$ \\
\hline Satellite & -25 degrees & & & & & & & - \\
\hline Satellite & 40 degrees & & & & & & & - \\
\hline Jamming & -6 degrees & $\bullet$ & $\bullet$ & $\bullet$ & & & & \\
\hline Jamming & 12 degrees & & $\bullet$ & $\bullet$ & & & & \\
\hline Jamming & 40 degrees & & & $\bullet$ & & & & \\
\hline Jamming & -14 degrees & & & & $\bullet$ & & & $\bullet$ \\
\hline Jamming & 12 degrees & & & & - & & & \\
\hline Jamming & 24 degrees & & & & & $\bullet$ & & - \\
\hline Jamming & -22 degrees & & & & & $\bullet$ & & - \\
\hline Jamming & 72 degrees & & & & & & $\bullet$ & \\
\hline Jamming & 0 degrees & & & & & & - & \\
\hline Jamming & -60 degrees & & & & & & & $\bullet$ \\
\hline
\end{tabular}

- indicates the selected satellite signal or jamming signal.

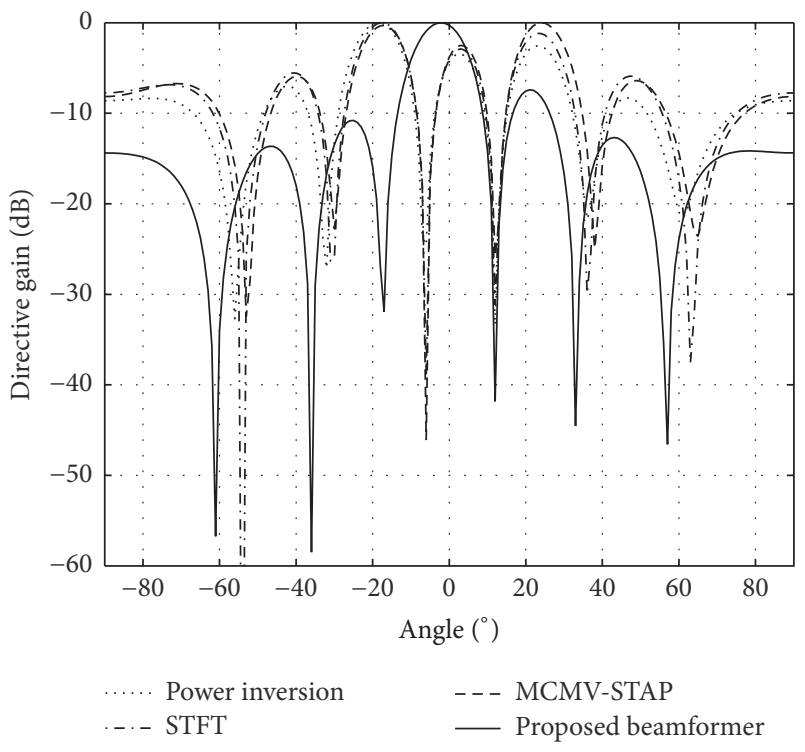

FIGURE 6: The array antenna pattern of antijamming for different method: the angle of arrival of the satellite signal is 0 degrees and the angles of arrival of jamming signals are -6 degrees and 12 degrees.

distorts terribly. The pattern's 0 -degree gain is $3 \mathrm{~dB}$ less than the pattern without jamming, which results in lower output SINR of satellite signal. The method proposed in this paper produces no jamming nulling at -6 degrees. And 12-degree jamming, producing 2-degree main-lobe deviation because of 12-degree nulling, belongs to side-lobe and produces nulling. However, gain of the pattern without jamming, which has a lower side-lobe, is merely $0.3 \mathrm{~dB}$ less than one of 0 -degree pattern.

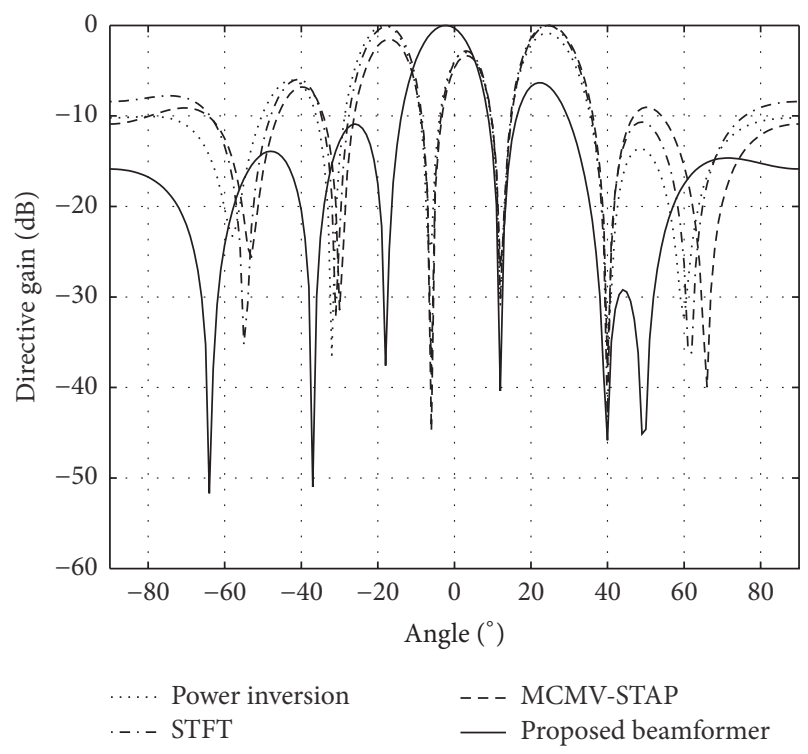

FIGURE 7: The array antenna pattern of antijamming for different method: the angle of arrival of the satellite signal is 0 degrees and the angles of arrival of jamming signals are -6 degrees, 12 degrees, and 40 degrees.

Figure 7 shows the simulation in the third scene of Table 1: presuming that incidence angle of satellite signal is 0 degrees. Incidence angles of jamming signal are -6 degrees, 12 degrees, and 40 degrees with an ISR of $40 \mathrm{~dB}$. The algorithms of minimum power, STFT, and MCMV-STAP can precisely produce nulling in jamming position, but the deviation of beam direction is bigger than the proposed method. And pattern's 0 -degree gain is $4.7 \mathrm{~dB}$ less than the pattern 


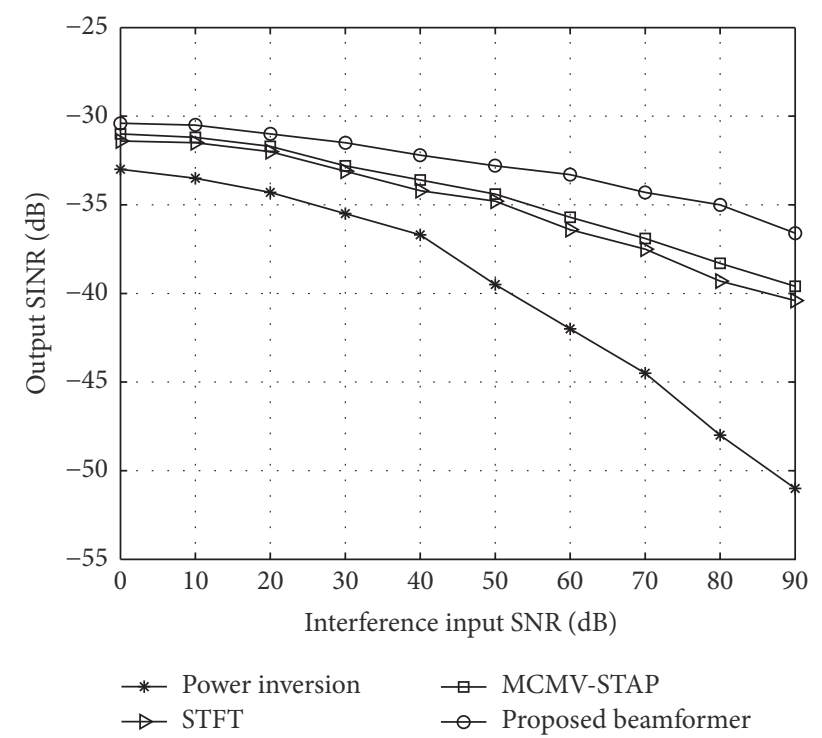

FIGURE 8: The output SINR of different method when suppress jamming: the angle of arrival of the satellite signal is 0 degrees and the angle of arrival of jamming signal is -6 degrees.

without jamming, and pattern's 0 -degree gain deteriorates even further. The method proposed in this paper produces jamming nulling at 12 degrees and 40 degrees. However, gain of the pattern without jamming, which has a lower side-lobe, is merely $0.3 \mathrm{~dB}$ less than one of 0 -degree pattern also.

Figures 5, 6, and 7 verify the validity of the method proposed in this paper. No nulling or deviation of beam direction appears when suppressing main-lobe jamming. This provides a solid guarantee for effectively receiving navigation signal since output SINR of navigation signal does not decrease because of beam-direction deviation.

4.2. Influence of Jamming-Signal Intensity on Output SINR of Satellite Signal. In this simulation experiment, seven-arrayelement antennas are evenly distributed with a distance of half wavelength width to each other, and ISR of jamming signal increases from $0 \mathrm{~dB}$ to $90 \mathrm{~dB}$. Influence of jammingsignal intensity on output SINR of satellite signal is analyzed under this circumstance.

Output SINR of navigation signal can be expressed as

$$
\operatorname{SINR}=\frac{\widehat{\mathbf{W}}^{H}{ }_{\text {LCMV }} \mathbf{R}_{s} \widehat{\mathbf{W}}_{\text {LCMV }}}{\widehat{\mathbf{W}}^{H}{ }_{\text {LCMV }}\left(\mathbf{R}_{i}+\mathbf{R}_{n}\right) \widehat{\mathbf{W}}_{\text {LCMV }}},
$$

among which $\mathbf{R}_{s}$ refers to sample covariance matrix and $\mathbf{R}_{i}+$ $\mathbf{R}_{n}$ refers to jamming-and-noise sample covariance matrix [29].

Figure 8 shows the simulation of the first scene in Table 1. Although the beam of minimum power algorithm in Figure 5 deviates 6 degrees, its output SINR curve is the worst because of its higher side-lobe electrical level. In the $0 \mathrm{~dB}$ to $40 \mathrm{~dB}$ interval, STFT algorithm and MCMV-STAP algorithm's performance is close. Yet MCMV-STAP one performs better than STFT one between $40 \mathrm{~dB}$ and $90 \mathrm{~dB}$. The reason is that

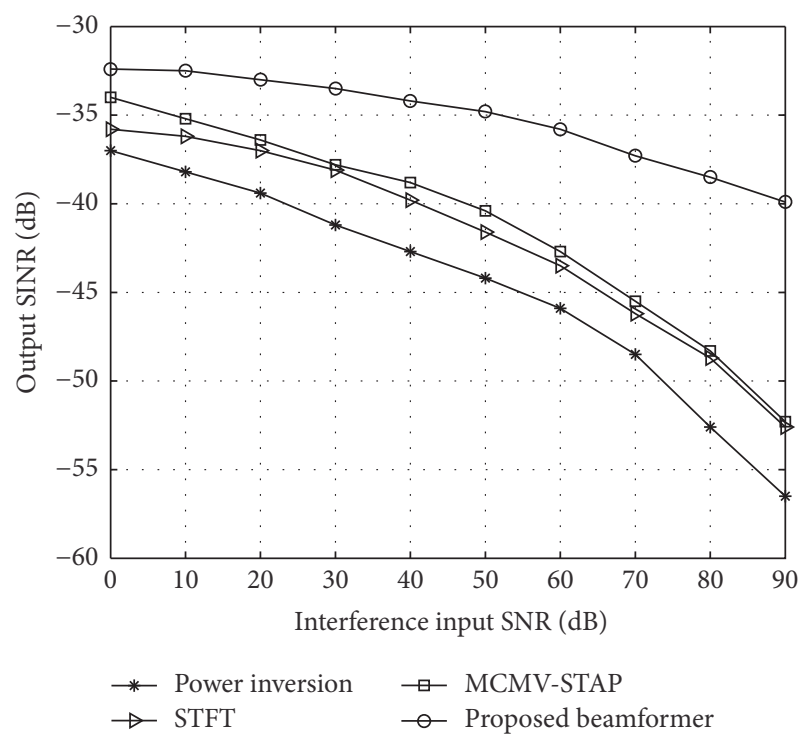

FIGURE 9: The output SINR of different method when suppress jamming: the angle of arrival of the satellite signal is 0 degrees and the angles of arrival of jamming signals are -6 degrees and 12 degrees.

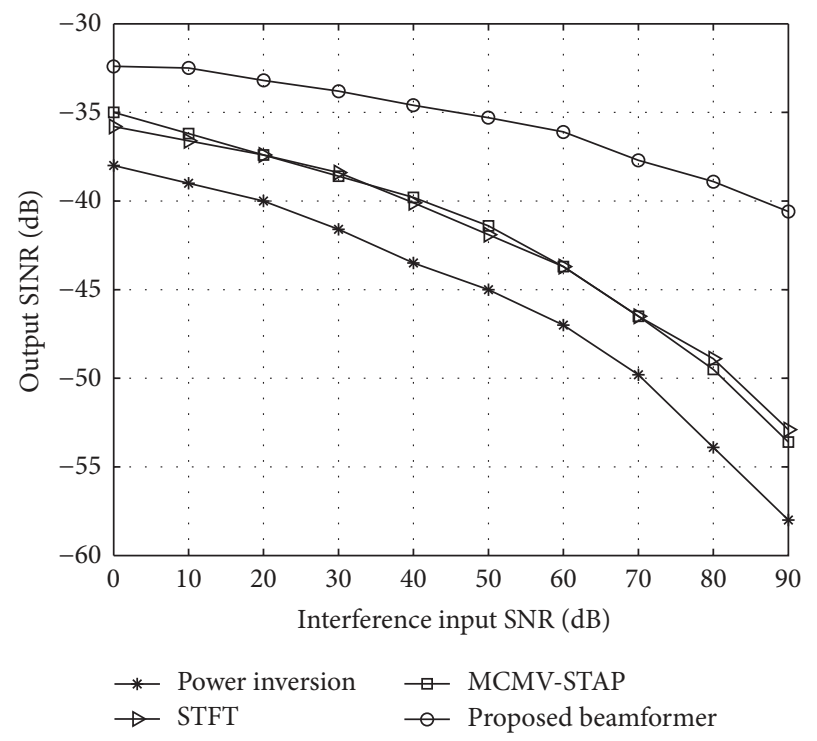

FIGURE 10: The output SINR of different method when suppress jamming: the angle of arrival of the satellite signal is 0 degrees and the angles of arrival of jamming signals are -6 degrees, 12 degrees, and 40 degrees.

MCMV-STAP algorithm utilizes multibeam as well as spacetime structure so that it constrains direction of navigation signal. The method proposed in this paper produces the best performance curve, since the pattern does not distort when suppressing main-lobe jamming.

Figure 9 shows the simulation of the second scene in Table 1, and Figure 10 shows the simulation in the third scene in Table 1. Apparently, the output SINR of navigation 


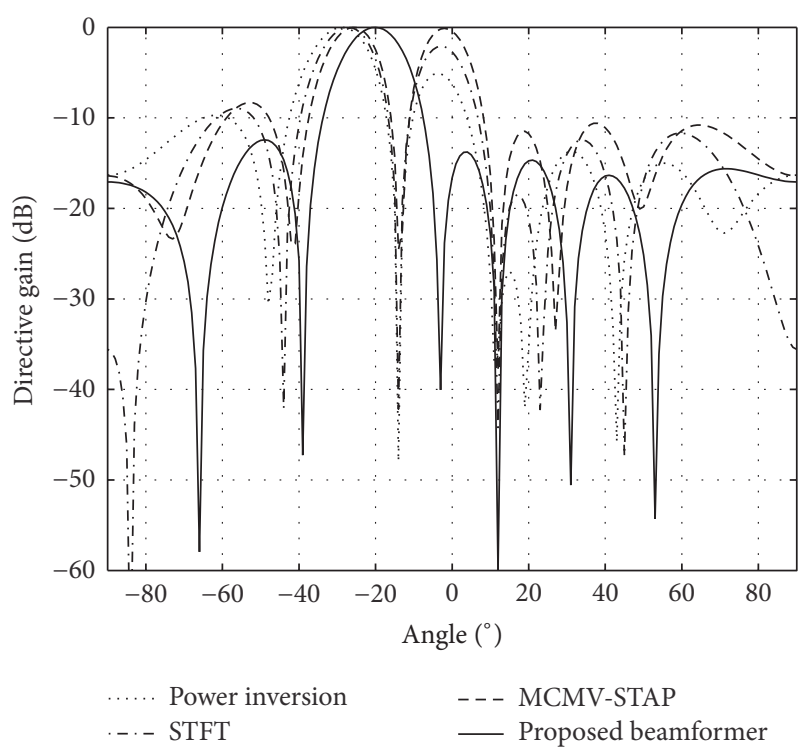

FIGURE 11: The array antenna pattern of antijamming for different method: the angle of arrival of the satellite signal is -20 degrees and the angles of arrival of jamming signals are -14 degrees and 12 degrees.

is the worst when minimum power algorithm imposes no constraint on direction of navigation signal. Having treated information about frequency domain, STFT algorithm performs equally with MCMV-STAP one in terms of output SINR. Under this circumstance, the method proposed in this paper has the best performance curve of output SINR during jamming suppression.

\subsection{Influence of Jamming Signal with Various Incidence Angles} on Pattern. This section focuses on influence of jamming signal with various incidence angles on the pattern. In this section, a seven-array-element ULA is evenly distributed with a distance of half wavelength width from each other. But ISR of jamming signal is a constant value of $50 \mathrm{~dB}$.

Figure 11 shows the simulation of the fourth scene in Table 1: incidence signal of navigation signal is -20 degrees and ones of jamming signals are -14 degrees and 12 degrees. Algorithms of STFT and MCMV-STAP form two sets of jamming nulling and beam-direction deviation. The method proposed in this paper produces nulling at 12-degree jamming but not at -14-degree main-lobe jamming. The mainlobe direction does not distort.

Figure 12 shows the simulation of the fifth scene in Table 1: incidence signal of navigation signal is 20 degrees and ones of jamming signals are 24 degrees and -22 degrees. Algorithms of STFT and MCMV-STAP form two sets of jamming nulling and beam-direction deviation. The method proposed in this paper produces nulling at -22-degree jamming but not at 24-degree main-lobe jamming. Main-lobe direction does not distort.

Figure 13 shows the simulation of the sixth scenario in Table 1: incidence signal of navigation signal is 70 degrees and ones of jamming signals are 72 degrees and 0 degrees.

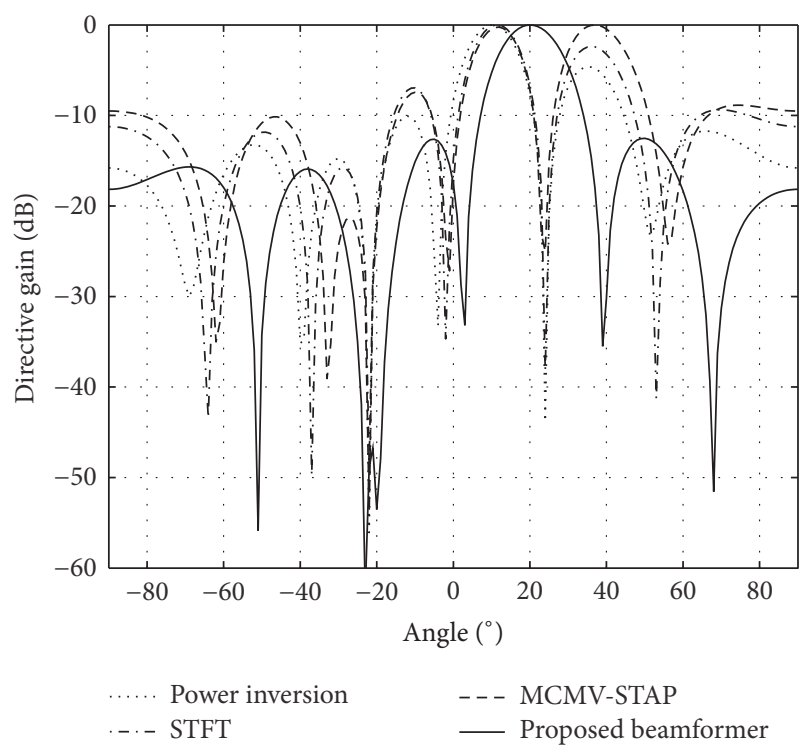

FIGURE 12: The array antenna pattern of antijamming for different method: the angle of arrival of the satellite signal is 20 degrees and the angles of arrival of jamming signals are 24 degrees and -22 degrees.

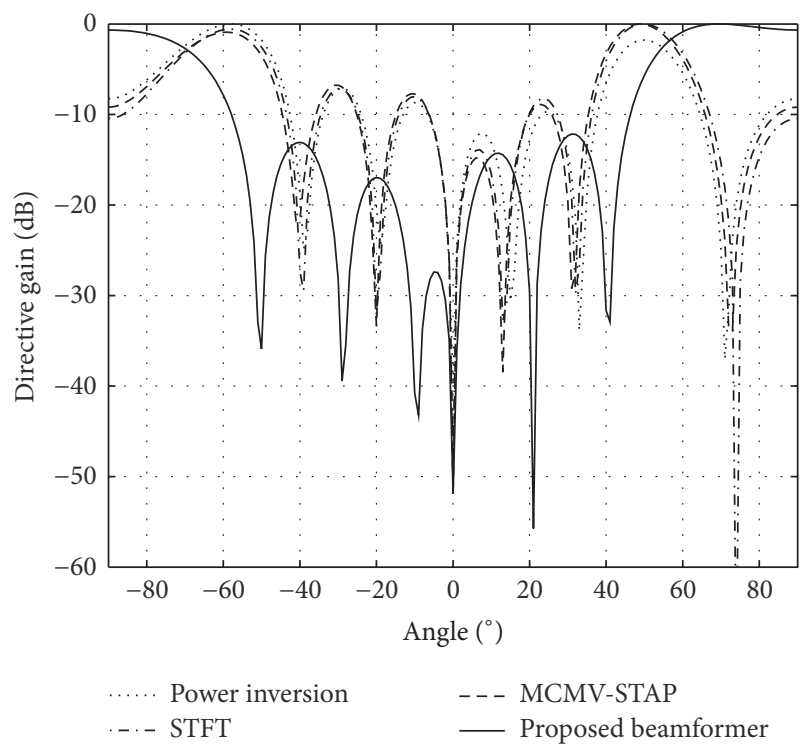

FIgURE 13: The array antenna pattern of antijamming for different method: the angle of arrival of the satellite signal is 70 degrees and the angles of arrival of jamming signals are 72 degrees and 0 degrees.

Algorithms of STFT and MCMV-STAP form two sets of jamming nulling and beam-direction deviation. The method proposed in this paper produces nulling at 0 -degree jamming but not at 72-degree main-lobe jamming. The main-lobe direction does not distort.

4.4. Influence of Jamming Signal among Multisatellite Navigation Signal on Pattern. This subsection simulates the seventh scenario in Table 1: incidence angles of navigation signals are 


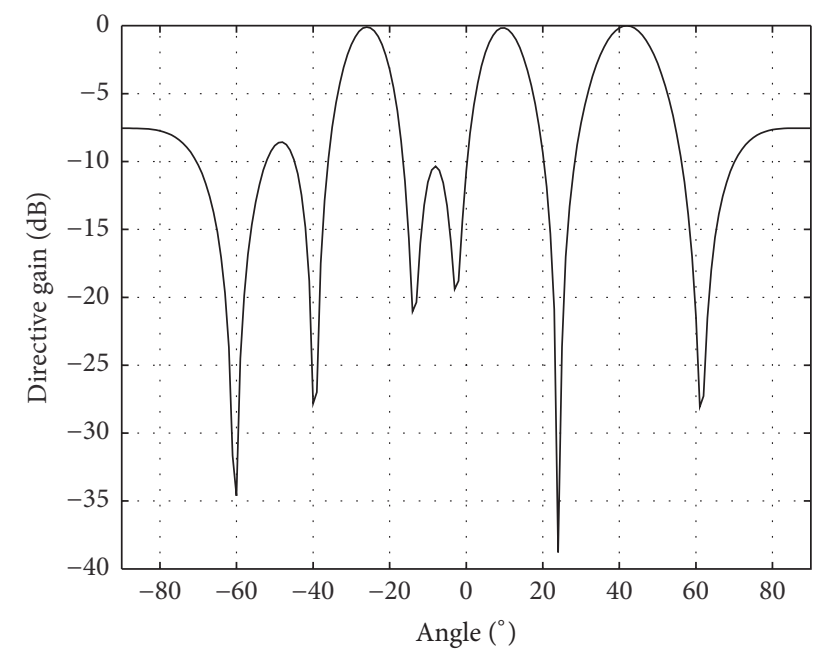

FIGURE 14: The array antenna pattern of the proposed method: the angles of arrival of the satellite signal are 10 degrees, -25 degrees, and 40 degrees and the angles of arrival of jamming signals are -14 degrees, -22 degrees, -60 degrees, and 24 degrees.

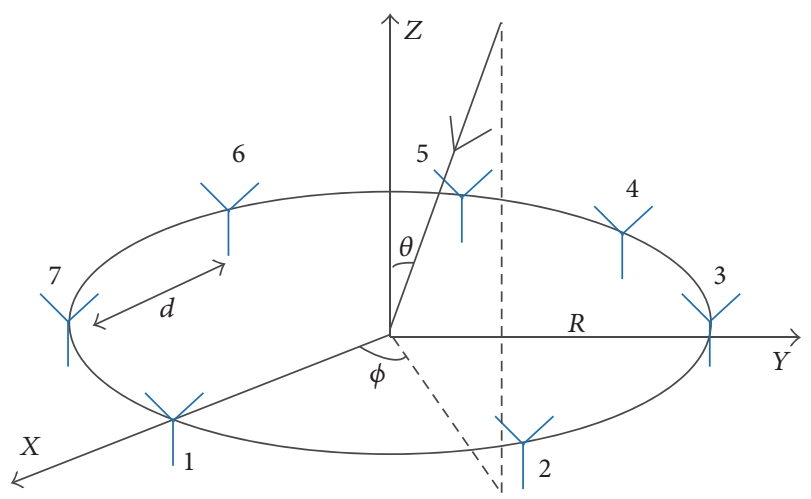

FIGURE 15: The seven-sensor uniform circular array geometry.

-25 degrees, 10 degrees, and 40 degrees. Ones of jamming signals are -60 degrees, -22 degrees, -14 degrees, and 24 degrees, among which the second one is at the mainlobe. LCMV beamformer produces three beams, pointing at incidence angles of three navigation signals relatively.

In Figure 14, seven-array-element aerials are evenly distributed with a distance of half wavelength width from each other, and ISR of jamming signal is $40 \mathrm{~dB}$, fixed. Jamming nulling only appears in adaptive pattern at -60 degrees, -14 degrees, and 24 degrees, yet none at -22 -degree main-lobe jamming. Furthermore, pattern direction does not distort, which is in favor of effectively receiving navigation signal.

\subsection{Influence of Jamming Signal on a Seven-Sensor Uniform} Circular Array Pattern. The method proposed in this paper is applied in the seven-sensor uniform circular array pattern (Figure 15): seven-array elements are evenly distributed on the circle with half wavelength (the array-element' distance is $d$ ) as radius $R$. Signal incidence angle is $(\theta, \phi)$; the former

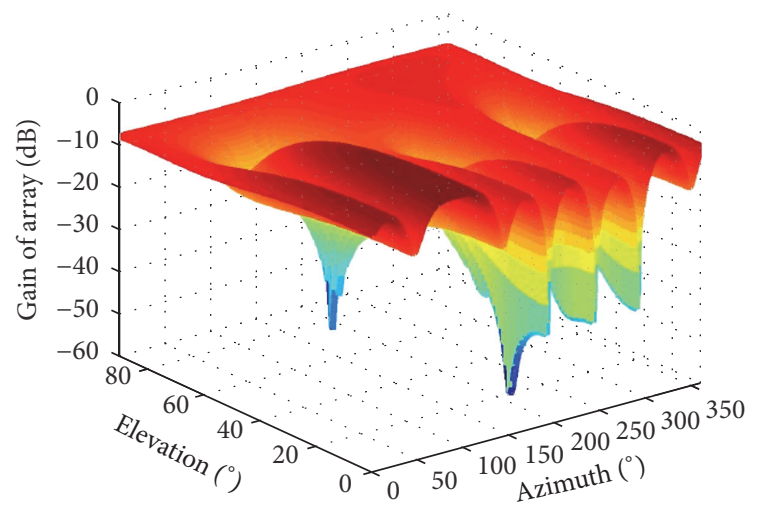

FIGURE 16: Beams and nulls 3D pattern of the proposed method according to the scenario in Table 2 and Jamming Scenario in Section 4.5.

one refers to azimuth and the latter to pitching angle [30]. So corresponding steering vector can be expressed as

$$
a(\theta, \phi)=\left[\begin{array}{c}
\exp \left[\frac{j 2 \pi R \sin \theta \cos \left(\phi-\gamma_{0}\right)}{\lambda}\right] \\
\exp \left[\frac{j 2 \pi R \sin \theta \cos \left(\phi-\gamma_{1}\right)}{\lambda}\right] \\
\vdots \\
\exp \left[\frac{j 2 \pi R \sin \theta \cos \left(\phi-\gamma_{M-1}\right)}{\lambda}\right]
\end{array}\right]
$$

among which $\gamma_{m}=2 \pi m / N(m=0,1, \ldots, 6)$.

In order to verify the validity of this method in real condition, the simulation in this subsection is implemented at 11 o'clock on 14 April, 2016, at $40^{\circ} \mathrm{N}, 108.45^{\circ} \mathrm{E}$. Table 2 shows the actual position of GPS satellite.

The following Jamming Scenario shows the parameters of jamming with respect to satellite's coordinate position in Table 2:

$$
\begin{aligned}
& \text { Four-CW jamming } \\
& \text { Azimuth }=81,146,201,342 \\
& \text { Elevation }=17,67,15,48
\end{aligned}
$$

The results of simulation by this method are shown by Figures 16 and 17; the former one is three-dimensional (3D) antenna pattern and the latter one shows two-dimensional (2D) direction and pitching angle. Meanwhile, Figure 17 illustrates positions of navigation signal and jamming: jamming 1 and jamming 2 are at main-lobe but form no nulling, while jamming 3 and jamming 4 form precise nulling. Navigation signals are at respective beam main-lobe, where no deviation appears. This conforms to the result of theoretical analysis.

\section{Conclusion}

On the basis of reconstructing sample covariance matrix, this paper proposes a method to suppress jamming on satellite navigation. No main-lobe nulling is formed by the proposed 
TABLE 2: Azimuth and elevation angles of visible satellites.

\begin{tabular}{|c|c|c|c|}
\hline PRN & $\mathrm{Az}$ (degree) & El (degree) & Sky plot \\
\hline 1 & 24 & 5 & $\begin{array}{ll}90 & 100 \\
\end{array}$ \\
\hline 4 & 98 & 10 & \\
\hline 10 & 170 & 19 & \\
\hline 12 & 221 & 6 & 20 \\
\hline 14 & 320 & 10 & $\therefore$ \\
\hline 18 & 112 & 39 & \\
\hline 22 & 99 & 35 & \\
\hline 25 & 270 & 62 & \\
\hline 32 & 312 & 81 & \\
\hline 33 & 58 & 52 & 270 \\
\hline
\end{tabular}

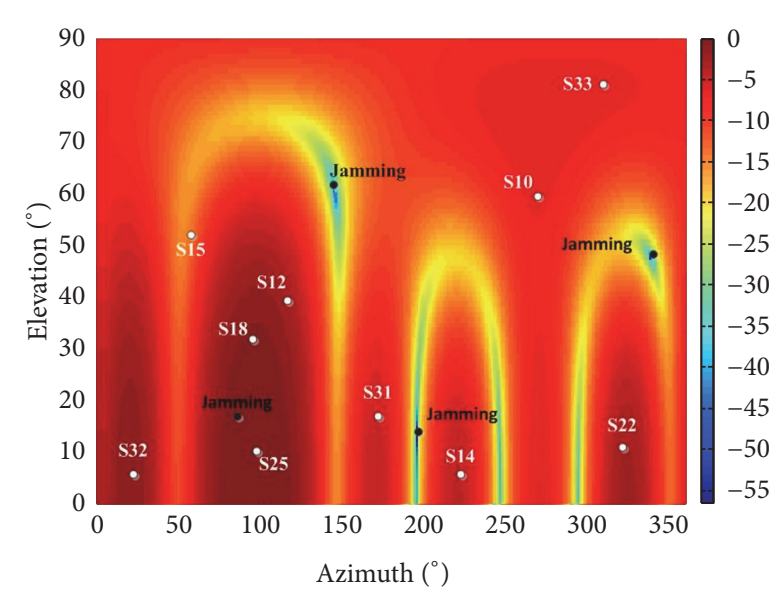

FIGURE 17: Beams and nulls 2D pattern of the proposed method according to the scenario in Table 2 and Jamming Scenario in Section 4.5.

method. Combined with APC, the adaptive pattern of array antenna approaches the pattern without jamming so as to receive the matching navigation signal.

This proposed method suppresses the jamming by constructing eigenvector matrix, because of the sampling covariance matrix reconstruction. The main-lobe jamming does not null. This pattern always accurately points to the direction of navigation satellite. The pattern accurately formed deep nulling at the side-lobe jamming. Compared with other methods, it has the highest output SINR. This is helpful for the navigation receiver to solve the navigation signal and can effectively improve the navigation system antijamming capacity.

Numerical simulation and case study results in this paper show that the pattern always precisely points to the direction of navigation satellite during jamming suppression. Deep nulling is achieved only on the position of side-lobe jamming. The proposed method has the highest output SINR compared with other methods. This is beneficial to the navigation signal receiving as well as improving the antijamming performance of the GNSS receiver.

\section{Competing Interests}

We declare that there is no conflict of interests regarding the publication of this paper.

\section{Acknowledgments}

This work has been supported by the National Natural Science Foundation of China (nos. 61271416, 61301093, and 61501376) and Fundamental Research Funds for the Central Universities (no. 3102016ZY020).

\section{References}

[1] T. Marathe, S. Daneshmand, and G. Lachapelle, "Assessment of measurement distortions in GNSS antenna array space-time processing," International Journal of Antennas and Propagation, vol. 2016, Article ID 2154763, 17 pages, 2016.

[2] G. Galati, R. Dellago, and F. Lanari, "Global navigation satellite system in an integrated air traffic management constellation," IEE Proceedings-Radar, Sonar and Navigation, vol. 144, no. 3, pp. 156-162, 1997.

[3] J. H. Blakey, "Navigating towards the future: transitioning from terrestrial radio navigation to satellite navigation and airborne surveillance," IEEE Aerospace and Electronic Systems Magazine, vol. 21, no. 5, pp. 17-21, 2006.

[4] A. Emmanuele, F. Zanier, G. Boccolini, and M. Luise, "Spreadspectrum continuous-phase-modulated signals for satellite navigation," IEEE Transactions on Aerospace and Electronic Systems, vol. 48, no. 4, pp. 3234-3249, 2012.

[5] A. R. Lopez, "GPS landing system reference antenna," IEEE Antennas and Propagation Magazine, vol. 52, no. 1, pp. 104-113, 2010.

[6] C. J. Wullems, "A spoofing detection method for civilian L1 GPS and the E1-B galileo safety of life service," IEEE Transactions on Aerospace and Electronic Systems, vol. 48, no. 4, pp. 2849-2864, 2012.

[7] W. Wang, Q. Du, R. Wu, and J. Liang, "Interference suppression with flat gain constraint for satellite navigation systems," IET Radar, Sonar and Navigation, vol. 9, no. 7, pp. 852-856, 2015.

[8] R. L. Fante and J. J. Vaccaro, "Wideband cancellation of interference in a GPS receive array," IEEE Transactions on Aerospace and Electronic Systems, vol. 36, no. 2, pp. 549-564, 2000. 
[9] W. L. Myrick, M. D. Zoltowski, and J. S. Goldstein, "Lowsample performance of reduced-rank power minimization based jammer suppression for GPS," in Proceedings of the IEEE 6th International Symposium on Spread Spectrum Techniques and Applications (ISSSTA '00), pp. 93-97, September 2000.

[10] W. Sun and M. G. Amin, "A self-coherence anti-jamming GPS receiver," IEEE Transactions on Signal Processing, vol. 53, no. 10, pp. 3910-3915, 2005.

[11] W. L. Myrick, G. J. Scott, and M. D. Zoltowski, "Low complexity anti-jam space-time processing for GPS," in Proceedings of the IEEE International Conference on Acoustics, Speech, and Signal Processing, pp. 2233-2236, Salt Lake City, Utah, USA, May 2001.

[12] M.-J. Yu, "INS/GPS integration system using adaptive filter for estimating measurement noise variance," IEEE Transactions on Aerospace and Electronic Systems, vol. 48, no. 2, pp. 1786-1792, 2012.

[13] M. J. Rezaei, M. Abedi, and M. R. Mosavi, "New GPS antijamming system based on multiple short-time Fourier transform," IET Radar, Sonar and Navigation, vol. 10, no. 4, pp. 807815, 2016.

[14] B. Motella, S. Savasta, D. Margaria, and F. Dovis, "Method for assessing the interference impact on GNSS receivers," IEEE Transactions on Aerospace and Electronic Systems, vol. 47, no. 2, pp. 1416-1432, 2011.

[15] A. Khabbazibasmenj, S. A. Vorobyov, and A. Hassanien, "Robust adaptive beamforming based on steering vector estimation with as little as possible prior information," IEEE Transactions on Signal Processing, vol. 60, no. 6, pp. 2974-2987, 2012.

[16] J. Zhuang and A. Manikas, "Interference cancellation beamforming robust to pointing errors," IET Signal Processing, vol. 7, no. 2, pp. 120-127, 2013.

[17] O. G. Vendik and D. S. Kozlov, "Phased antenna array with a side-lobe cancellation for suppression of jamming," IEEE Antennas and Wireless Propagation Letters, vol. 11, pp. 648-650, 2012.

[18] X. Yang, P. Yin, T. Zeng, and T. K. Sarkar, "Applying auxiliary array to suppress mainlobe interference for ground-based radar," IEEE Antennas and Wireless Propagation Letters, vol. 12, pp. 433-436, 2013.

[19] Q. Li, W. Wang, D. Xu, and X. Wang, "A robust anti-jamming navigation receiver with antenna array and GPS/SINS," IEEE Communications Letters, vol. 18, no. 3, pp. 467-470, 2014.

[20] C. Rosetti and C. Carnebianca, "NAVSAT: a global satellite based navigation system," IEEE Aerospace and Electronic Systems Magazine, vol. 2, no. 12, pp. 15-22, 1987.

[21] Y. Li, S. Gu, and N. Zheng, "MIMO radar transmit beampattern design for DOA estimation with sidelobe suppression," International Journal of Antennas and Propagation, vol. 2016, Article ID 1512843, 10 pages, 2016.

[22] S. Daneshmand, A. J. Jahromi, A. Broumandan, and G. Lachapelle, "GNSS space-time interference mitigation and attitude determination in the presence of interference signals," Sensors, vol. 15, no. 6, pp. 12180-12204, 2015.

[23] S. Daneshmand, N. Sokhandan, M. Zaeri-Amirani, and G. Lachapelle, "Precise calibration of a GNSS antenna array for adaptive beamforming applications," Sensors, vol. 14, no. 6, pp. 9669-9691, 2014.

[24] B. Bonet, I. Alcantarilla, D. Flament, C. Rodríguez, and N. Zarraoa, "The benefits of multi-constellation GNSS: reaching up even to single constellation GNSS users," in Proceedings of the 22nd International Technical Meeting of The Satellite Division of the Institute of Navigation (ION GNSS '09), pp. 1268-1280, Savannah, Ga, USA, September 2009.

[25] H. Dai, X. Wang, Y. Li, Y. Liu, and S. Xiao, "Main-lobe jamming suppression method of using spatial polarization characteristics of antennas," IEEE Transactions on Aerospace and Electronic Systems, vol. 48, no. 3, pp. 2167-2179, 2012.

[26] M. R. Mosavi and F. Shafiee, "Narrowband interference suppression for GPS navigation using neural networks," GPS Solutions, vol. 20, no. 3, pp. 341-351, 2016.

[27] R. L. Fante and J. J. Vacarro, "Cancellation of jammers and jammer multipath in a GPS receiver," IEEE Aerospace and Electronic Systems Magazine, vol. 13, no. 11, pp. 25-28, 1998.

[28] M. Meurer, A. Konovaltsev, M. Cuntz, and C. Hättich, "Robust joint multi-antenna spoofing detection and attitude estimation using direction assisted multiple hypotheses RAIM," in Proceedings of the 25th International Technical Meeting of the Satellite Division of the Institute of Navigation (ION GNSS '12), pp. 30073016, ION, Nashville, Tenn, USA, September 2012.

[29] A. J. O'Brien and I. J. Gupta, "Comparison of output SINR and receiver $\mathrm{C} / \mathrm{N}_{0}$ for GNSS adaptive antennas," IEEE Transactions on Aerospace and Electronic Systems, vol. 45, no. 4, pp. 1630$1640,2009$.

[30] K. Yang, Z. Zhao, and Q. H. Liu, "Fast pencil beam pattern synthesis of large unequally spaced antenna arrays," IEEE Transaction on Antennas and Propagation, vol. 61, no. 2, pp. 627-634, 2013. 


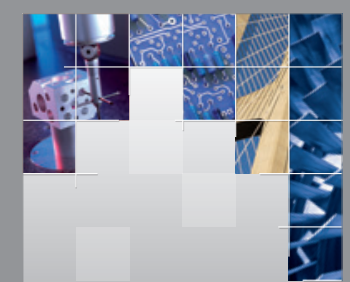

\section{Enfincering}
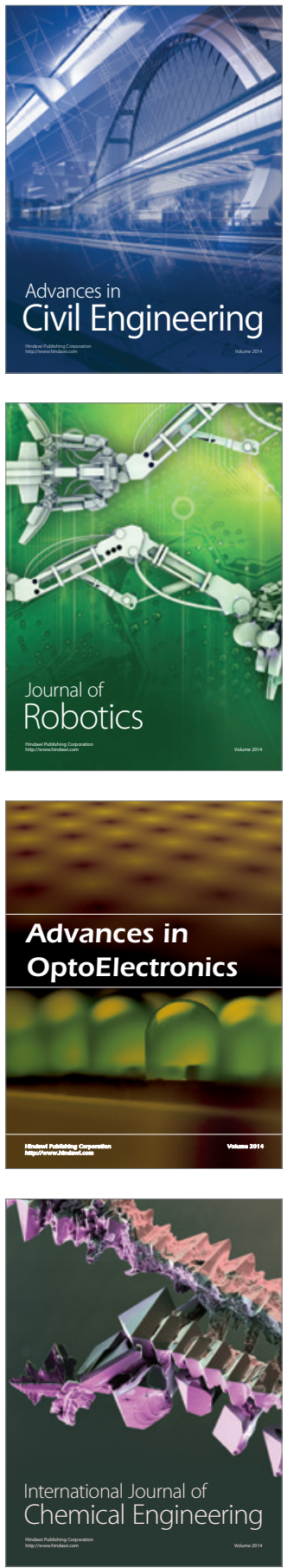

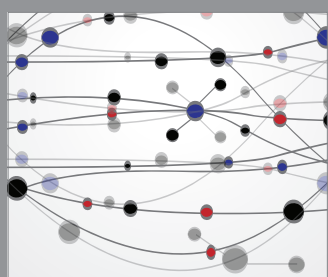

The Scientific World Journal

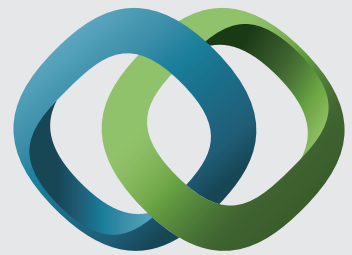

\section{Hindawi}

Submit your manuscripts at

https://www.hindawi.com
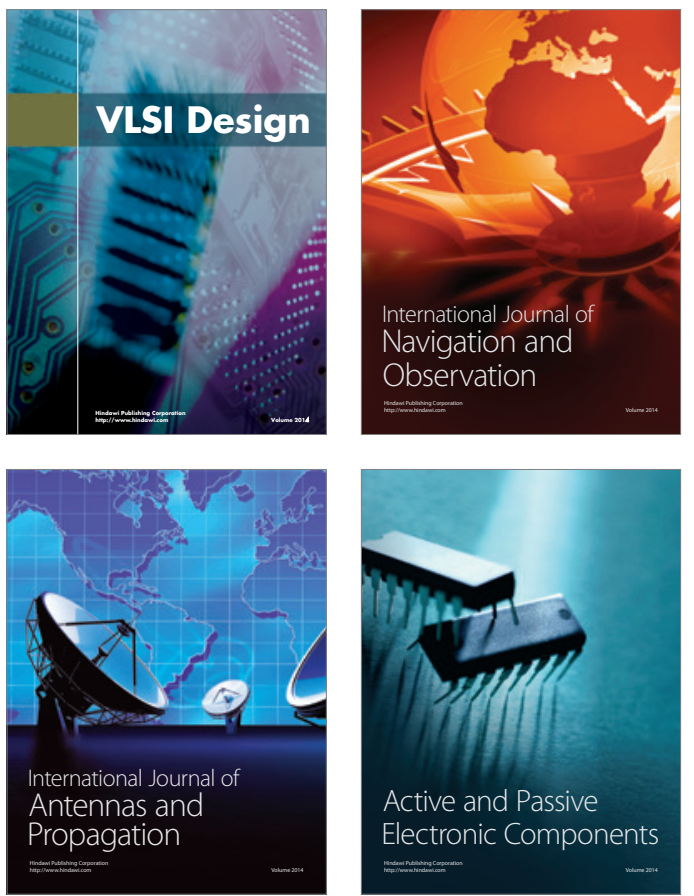
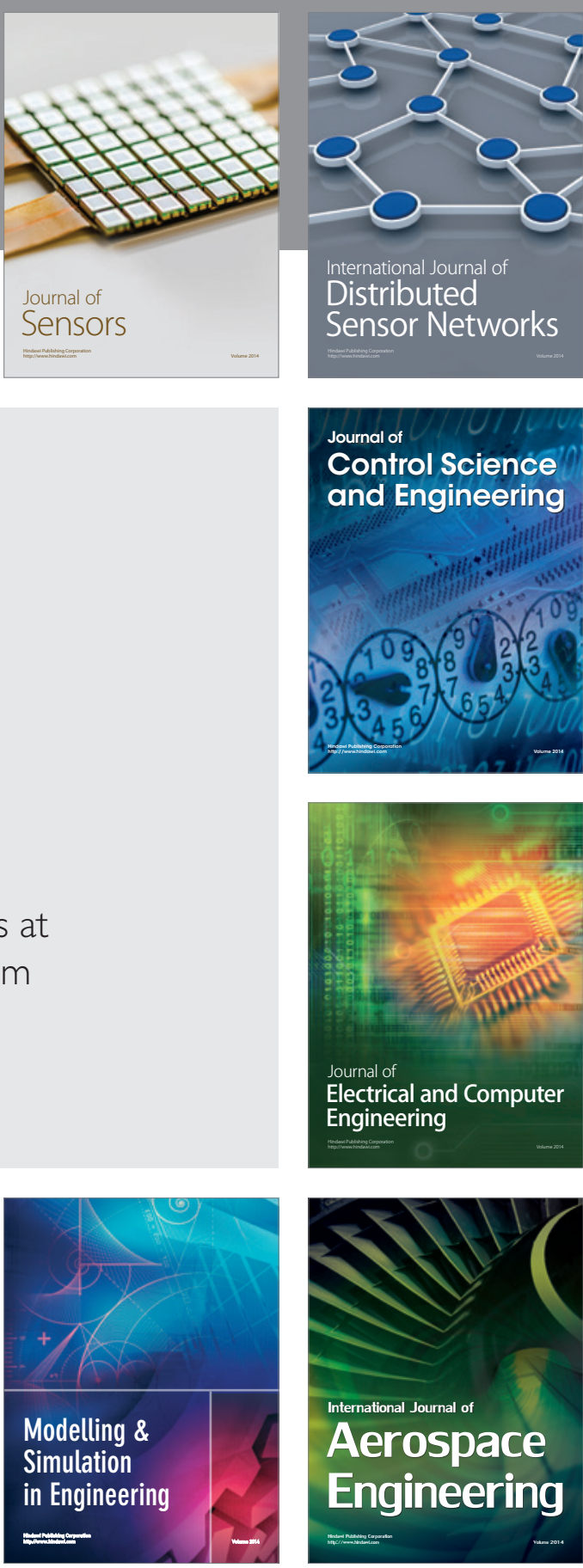

International Journal of

Distributed

Sensor Networks

$-$

Joumal of

Control Science

and Engineering
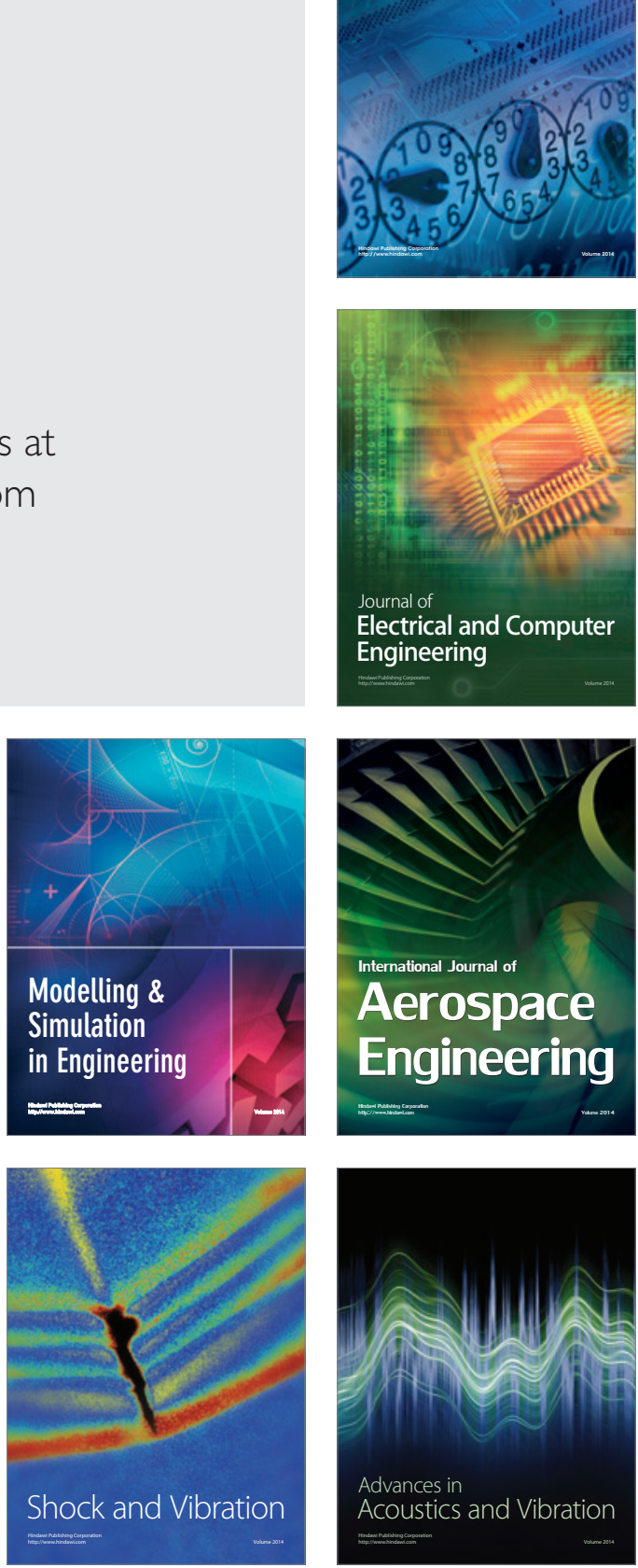